\title{
Dabigatran for intra-cardiac thrombus; Yet another promising role for novel oral anti-coagulants: A case report
}

\author{
Suresh Krishnamoorthy ${ }^{*}$, Tahir Ahmad ${ }^{2}$, Mahdi Halim ${ }^{1}$ and Asok Venkataraman ${ }^{1}$ \\ ${ }^{1}$ Consultant in Cardiology, George Eliot Hospital, United Kingdom \\ ${ }^{2}$ Consultant in Acute Medicine, George Eliot Hospital, United Kingdom
}

\begin{abstract}
Until recently, warfarin has been the main stay of treatment for thrombo-prophylaxis in atrial fibrillation (AF) patients. Indeed, with the emergence of new novel oral anticoagulants (NOACs) unsurprisingly there has been a noteworthy switch in the choice of antithrombotic treatment of AF patients. Nevertheless, still warfarin appears to be the drug of choice in patients with intra-cardiac thrombus (either left atrial thrombus or left ventricular mural thrombus). We report, a case of middle aged patient with recent onset atrial fibrillation and left atrial appendage thrombus with subsequent complete dissolution of thrombus following dabigatran treatment for 6-weeks. Despite, the promising results from limited case reports with NOACs, its efficacy and safety in the treatment of intra-cardiac thrombus needs to be evaluated in large randomised controlled trials.
\end{abstract}

\section{Case report}

A 54-year old middle-aged Caucasian gentleman with no significant past medical history was admitted with progressive breathlessness over a period of 2-3 months associated with intermittent palpitations. He was found to be in rapidly conducted atrial fibrillation at a rate of 160 bpm with signs of heart failure without any haemodynamic instability (Figure 1). He denies any excessive alcohol intake or any illicit drug abuse. There was no family history related to AF or any forms of cardiomyopathy. His echocardiogram revealed moderately dilated left ventricle with evidence of severe left ventricular dysfunction, thought to be probably rate-related; however with a large left atrial appendage (LAA) thrombus in a moderately dilated left atrium (LA) (Figure 2). There were no significant valvular abnormalities seen. His contrast computed tomography (CT) of thorax excluded any pulmonary embolism, but demonstrated presence of large LAA thrombus (Figure 3). He was started on all conventional heart failure treatment including loop diuretics, angiotensin converting enzyme inhibitor and beta blockers towards optimal ventricular rate control for his AF. His warfarin was switched to dabigatran $150 \mathrm{mg}$ twice daily for thrombo-prophylaxis (as normal renal functions) within view of drug induced rash, labile INR as well as patient's preference towards

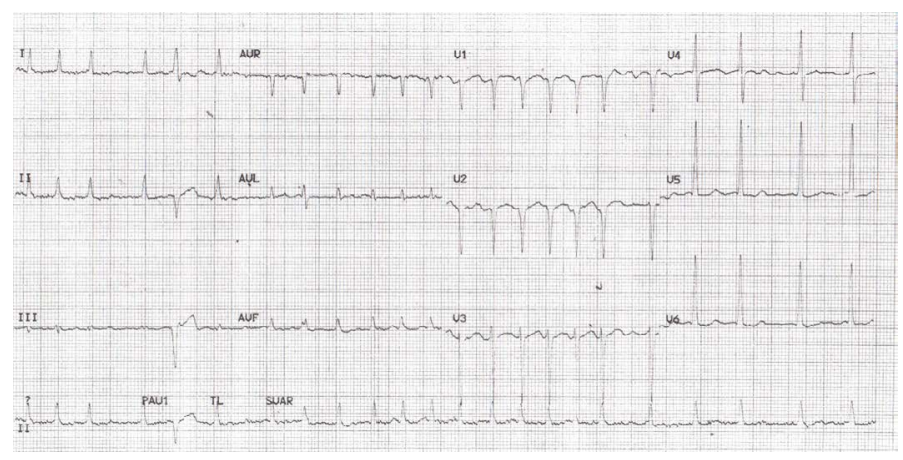

Figure 1. ECG confirming underlying rapidly conducted atrial fibrillation.
NOACs; however cautiously evaluating the risks and benefits. The rest of cardiomyopathy screen was negative for other reversible causes. His subsequent cardiac magnetic resonance imaging scan at 6-weeks later revealed complete dissolution of LAA thrombus and with slight improvement in his LV systolic function with optimal rate control (Figure 4). But there was no evidence of any infarction or fibrosis seen in the scan. He has dominant left coronary system with no flow obstructive lesions in his coronary tree. As he remained breathlessness

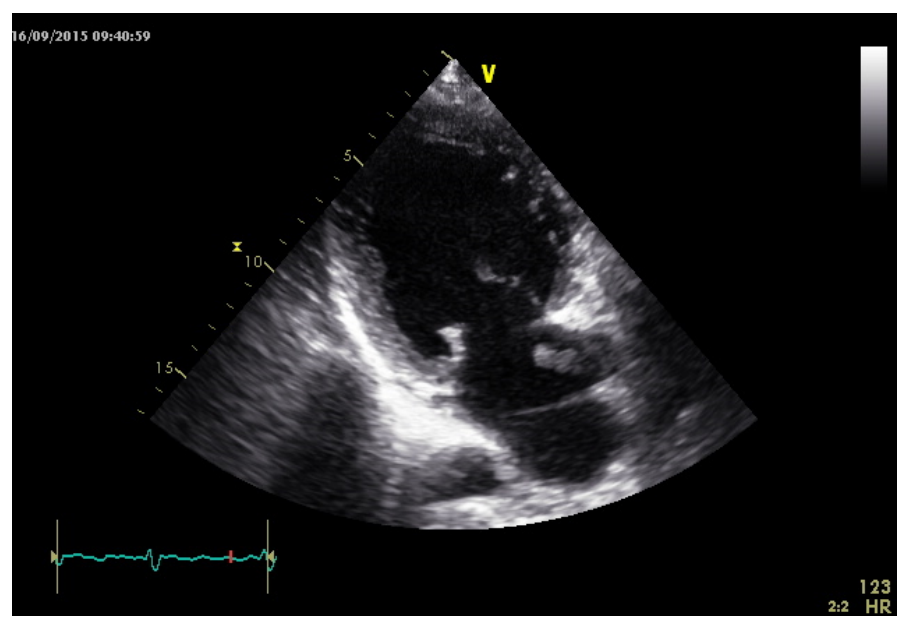

Figure 2. Trans-thoracic echocardiogram revealing dilated LV with the presence of left atrial thrombus.

Correspondence to: Suresh Krishnamoorthy, Consultant in Cardiology, George Eliot Hospital, College Road, Nuneaton, CV10 7DJ, United Kingdom, Tel: 02476 865 378, Fax: 02476 865704, E-mail: suresh.krishnamoorthy@geh.nhs.uk

Key words: intra-cardiac thrombus, left atrial thrombus, dabigatran

Received: November 01, 2016; Accepted: November 18, 2016; Published: November 21, 2016 


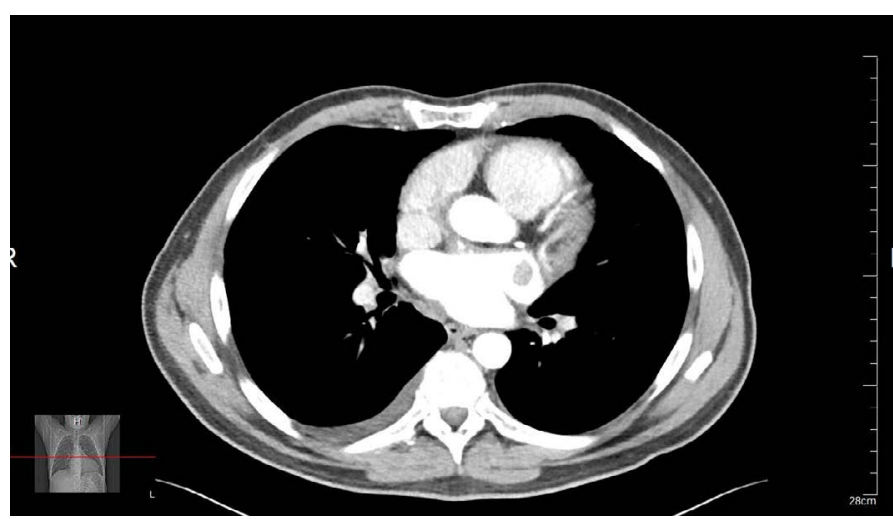

Figure 3. Contrast CT thorax revealing the presence of left atrial appendage thrombus.

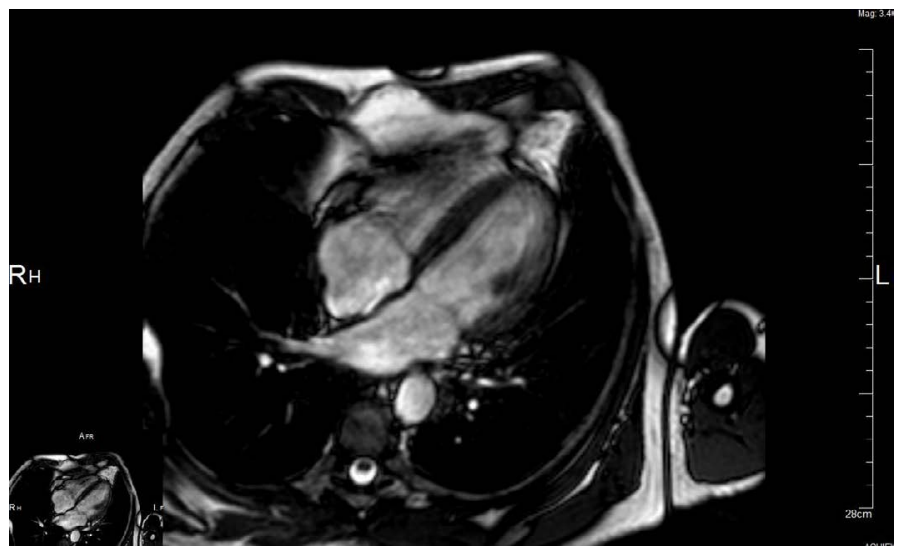

Figure 4. Cardiac MRI revealing the complete resolution of left atrial appendage thrombus with 6-weeks treatment with dabigatran.

despite adequate ventricular rate control, he had successful outpatient DC-cardioversion restoring sinus rhythm guided by trans-oesophageal echocardiogram (which once again revealed no evidence of LA or LAA thrombus). Notably, since restoring sinus rhythm he is doing well with significantly improved effort tolerance and less breathless; also his repeat echocardiogram demonstrated near normalisation of his LV systolic function. Nonetheless, we opted to continue his conventional heart failure treatment for now as well as his dabigatran, within view of re-evaluating things in the near future.

\section{Discussion}

There are enough compelling evidence(s) to suggest that AF renders pro-thrombotic state, via alterations in the individual components of "Virchow' triad" (blood stasis, cardiac structure and blood constituents). Indeed, the thrombo-embolic risk associated with AF remains heterogeneous and are often associated with co-existent comorbidities. AF patients should be individually risk assessed towards both thrombo-embolism and bleeding from anticoagulation. Further improvement in the thrombo-embolic risk stratification schemata with $\mathrm{CHA}_{2} \mathrm{DS}_{2}$-VASc Score [1] have helped clinicians to truly assess those at low-risk compared to moderate/high-risk patients. Until, recently vitamin-K antagonists (predominantly warfarin) were widely used for thrombo-prophylaxis in these AF patients. However, the emergence of newer novel oral anticoagulants (NOACs) has invariably assisted clinicians to sort an alternative to warfarin given their reliable form of antithrombotic effect, need for less monitoring as well as with minimal drug to drug and food interactions. At present the only major limitation with NOACs is the lack of specific anti-dote for individual agents. Nevertheless, there are ongoing pharmacological researches towards identifying specific anti-dotes for these individual novel oral anticoagulants and the preliminary results are quite promising.

Also, the evidences behind the role of NOACs in patients with intra-cardiac thrombus are quite sparse. Dabigatran etexilate is a pro-drug, and its active component "dabigatran" is an oral reversible, competitive direct thrombin inhibitor, which specifically binds to both free as well as clot-bound active thrombin molecules; thereby acts as a potent inhibitor of platelet aggregation. Morita, et al. [2] discussed the possible indirect thrombolytic effect of dabigatran in a patient with LA thrombus with complete resolution of thrombus following treatment with dabigatran $300 \mathrm{mg} /$ day for 4 -months as well as its potential in the treatment of these patients. Similarly, Nagamoto, et al. [3] in his case report demonstrated complete resolution of LV mural thrombus due to old anterior infarct with dabigatran $220 \mathrm{mg}$ /day for 4-weeks. Kaku, et al. [4] reported complete dissolution of thrombus following 3-weeks treatment with dabigatran $150 \mathrm{mg}$ twice daily in a hypertrophic cardiomyopathy patient with LV mural thrombus in an apical aneurysm. In a small study, up to $9 \%$ of patients with AF found to have LA thrombus on TEE with sub-therapeutic anticoagulation and associated with adverse outcomes [5]. At present, there are no specific guidelines in the treatment of patients with LA or LAA thrombus as mostly they found to be co-existent in patients with AF patients [6] or mitral stenosis. Nevertheless, in patients with LV mural thrombus, ESC guidelines [7] recommend vitamin-K antagonist for at least 3-6 months; whereas ACC/AHA guidelines [8] recommend indefinitely in patients those with low risk of bleeding. Clearly with the availability of even more potent dual antiplatelet and percutaneous reperfusion therapies, we are unfortunately relying on the remote ancient old data from the pre-thrombolytic and thrombolytic era in making decisions regarding the treatment of mural thrombus without any clear consensus.

\section{Conclusion}

Despite our exhaustive experience with warfarin over the years, truly we all appreciate and understand the unreliability of the drug in the real world practice and the absolute need for regular monitoring as well as its established interactions. Nonetheless, with the evolving experience with NOACs and individual case reports including ours, we believe there may be a role for NOACs even in patients with intracardiac thrombus. Without any doubt, the true efficacy and safety of the NOACs in the treatment of these patients with intra-cardiac thrombus needs to be evaluated in larger randomised controlled trials head-to-head with warfarin per se.

\section{Conflicts of interest}

The author(s) declare that there is no conflict of interest regarding the publication of this case report.

\section{References}

1. Lip GY, Nieuwlaat R, Pisters R, Lane DA, Crijns HJ (2010) Refining clinical risk stratification for predicting stroke and thromboembolism in atrial fibrillation using a novel risk factor-based approach: the euro heart survey on atrial fibrillation. Chest 137 : 263-272. [Crossref]

2. Morita S, Ajiro Y, Uchida Y, Iwade K (2013) Dabigatran for left atrial thrombus. Eur Heart J 34: 2745. [Crossref]

3. Nagamoto Y, Shiomi T, Matsuura T, Okahara A, Takegami K, et al. (2014) Resolution of a left ventricular thrombus by the thrombolytic action of dabigatran. Heart Vessels 29: 560-562. [Crossref]

4. Kaku B (2013) Intra-cardiac thrombus resolution after anti-coagulation therapy with dabigatran in a patient with mid-ventricular obstructive hypertrophic cardiomyopathy: 
A case report. J Med Case Rep 7: 238. [Crossref]

5. Fukuda S, Watanabe H, Shimada K, Aikawa M, Kono Y, et al. (2011) Left atria thrombus and prognosis after anticoagulation therapy in patients with atrial fibrillation. J Cardiol 58: 266-277. [Crossref]

6. January CT, Wann LS, Alpert JS, Calkins H, Cigarroa JE, et al. (2014) American College of Cardiology/American Heart Association Task Force on Practice Guidelines. $2014 \mathrm{AHA} / \mathrm{ACC} / \mathrm{HRS}$ guideline for the management of patients with atrial fibrillation: a report of the American College of Cardiology/American Heart Association Task Force on Practice Guidelines and the Heart Rhythm Society. J Am Coll Cardiol 64: e1-76.
7. Task Force on the management of ST-segment elevation acute myocardial infarction of the European Society of Cardiology (ESC), Steg PG, James SK, Atar D, Badano LP, et al. (2012) ESC Guidelines for the management of acute myocardial infarction in patients presenting with ST-segment elevation. Eur Heart J 33: 2569-2619. [Crossref]

8. O'Gara PT, Kushner FG, Ascheim DD, Casey DE Jr, Chung MK, et al. (2013) 2013 ACCF/AHA guideline for the management of ST-elevation myocardial infarction: executive summary: a report of the American College of Cardiology Foundation/ American Heart Association Task Force on Practice Guidelines: developed in collaboration with the American College of Emergency Physicians and Society for Cardiovascular Angiography and Interventions. Catheter Cardiovasc Interv 82: E1-27. [Crossref]

Copyright: (C2016 Krishnamoorthy S. This is an open-access article distributed under the terms of the Creative Commons Attribution License, which permits unrestricted use, distribution, and reproduction in any medium, provided the original author and source are credited. 